\title{
Yoga in school-aged children: Impact on children's mental health and well-being during the COVID-19 pandemic - A protocol
}

\begin{abstract}
The rapid spread of COVID-19 and the increasing burden on mental health and well- being require new ways of supporting families that have to maintain the new norms of social distancing. Many families may be under enormous amounts of stress and anxiety caused by losing their loved ones. Therefore, there is a great need to search for ways to minimize the effects of COVID-19 on mental health and well-being. The purpose of the research is to evaluate a yoga intervention offered online to school-aged children in the U.K. by Patanjali Yog Peeth Trust U.K. (PYPT) and evaluate its effectiveness by interviewing the parents of participating children. The project will seek to promote an additional tool in managing mental health and well-being in school-aged children. The research will use a mixed-methods approach. Parents will be approached by a PYPT yoga teacher and invited to complete an online survey and quantitative interview. Parents (over 18 years) of school-aged children (5-16 years) who live in the U.K. are eligible to take part. Upon gaining consent, participates will be asked to complete a parent and child characteristic questionnaire. An SDQ survey for parents via online Opinio and a subsample will be interviewed using a structured interview schedule. All analyses will be carried out in SPSS. It is intended that the research will provide an evidence base that will aid future studies and inform in developing yoga interventions that may aid in promoting child mental health and well-being. The study results will be published in peer-reviewed international journals; the researchers will also present the results at governmental conferences.
\end{abstract}

Keywords: Adolescent - Anxiety - Children - Child - Childhood - COVID-19 - Depression Mental health - Mindfulness - School - Stress - Well-being - Yoga

\section{Introduction}

The early years of a child's development are often perceived as carefree and removed from daily hassles, societal pressures, and systemic influences ${ }^{[1]}$. There is a common tendency for parents and caregivers to believe that children are immune to experience stress. $\left.{ }^{2}, 3\right]$, The current Healthy Child Programme (HCP) government policy $[4,5]$ encourages early intervention tackling mental health and well-being. According to Sheridan (2014), [6] early childhood development includes many tasks such as forming attachments, acquiring independence, developing their individuality, learning to play with peers, establishing values and mastering new skills. Additionally, parental relationships, family dynamics, socio-economic status and health status all influence a child's well-being and development.

Increasingly, school children present with challenging emotional and behavioural problems and may be resistant to traditional special education approaches ${ }^{[7]}$. Children's mental health and wellbeing have been known to lead to poor academic performance and behavioural problems both at home and in the school environment ${ }^{[8]}$. Children suffering from mental health illness often have other associated problems, such as anxiety, depression, and learning disabilities. As these children reach adolescence, they are also at a greater risk of drug and alcohol use. Children with these conditions are also known to suffer from higher temper tantrum levels and have difficulties with family and peer relationships. ${ }^{[8,9]}$ If mental health illnesses remain untreated in a child, it can 
continue into adulthood, preventing them from achieving their maximum potential.[10, 11] research suggests that yoga may increase mental health and well-being, positive behaviours, and aid learning skills in children and adolescents. [12]

Yoga is a holistic approach to health promotion that focuses on the interconnectedness of the body, mind, and spirit. ${ }^{[13]}$ It is a combination of physical poses (called Asanas), breathing techniques (called Pranayama), meditation, and relaxation. ${ }^{[14]}$ Yoga is becoming increasingly popular as a health promotion intervention in many parts of the world. Therefore, clinicians are turning to yoga as an intervention in the paediatric population. ${ }^{[15]}$ In its simplest form, yoga is a combination of controlled breathing (Pranayama) and different poses (Asanas). The essential part of any yoga pose is pranayama breathing in and out through the nose. Pranayama circulates more oxygen into the lungs and reduces stress, whereas breathing from the mouth can lead to hyperventilation and cause or worsen health. ${ }^{[16]}$ By practising deep relaxation or yoga breathing, cortisol activities in the nervous and endocrine systems are balanced, reducing the body's reaction to stress. As a result, the body produces fewer stress hormones (adrenaline, noradrenaline and cortisol) and becomes calm and at peace ${ }^{[1]}$; reduction in the levels of the stress hormone cortisol promotes sleep and encourages positive interactions ${ }^{[17]}$. Research suggests that yoga is used in various ways within the paediatric population17; for example, it has been used to aid in regulating emotions, facilitated positive self-esteem, and used to support a child's physical well-being. According to the Centers for Disease Control and Prevention (CDC) (2016), ${ }^{[18]}$ motor development activities as yoga can aid in child development, better body awareness, self-control, flexibility, and coordination. Bazzano et al. (2018) ${ }^{[19]}$ argue that learning yoga can help improve a child's skills that can be applied to many other activities. Children who focus on pranayama breathing have been noted to feel calm and less anxious before taking tests. [20]

Physical inactivity and unhealthy eating patterns are also another cause of concern for the National Health Service (NHS) services. According to the World Health Organisation (WHO) (2015), [21] children must participate in at least 60 minutes of physical activity every day. Practising yoga may be one way children can meet this guideline. Children who are overweight or obese are at the forefront of developing other mental health conditions like depression, anxiety, low selfesteem, suicide, substance misuse, self-harm resulting in lower educational and employment achievement. [22, 23] According to NHS (2017) ${ }^{[22]}$ figures, 1 in 8 children are affected by some form of mental health, which costs the health service $£ 3$ billion every year. Local areas spend $£ 54$ per child on mental health, compared to $£ 800$ on physical health. These figures illustrate the need to address childhood mental health.

\section{The impact of Covid-19 on child mental health}

Children whose parents have poor mental health are much more likely to have a mental health problem. A longitudinal survey by the NHS showed that the increase in possible mental health problems reported in children aged 5-16 years in England has risen from 10.8\% in 2017 to $16.0 \%$ in July 2020 across age, gender, and ethnic groups. ${ }^{24]}$ The rapid spread of COVID-19 and the increasing burden on mental health and well-being have led us to look at ways to support families that have been forced into maintaining the new norms of social distancing. Many families are under enormous amounts of stress and anxiety caused by the loss of their loved ones. ${ }^{[9]}$ The Office for National Statistics $(2020)^{[25]}$ has highlighted that ethnic minorities have a heightened risk of death from COVID-19. 
A range of risk factors for poor mental health has substantially increased amongst young children during the COVID-19 pandemic, such as loss of routine, reduced access to outdoor space, social isolation, relationship problems, family bereavement, concerns about education, future opportunities and health anxieties. [9] Schools provide access to many protective factors for children, including routine, strong peer support, and mental health support and engagement in physical activity have all been temporarily lost or reduced due to the current pandemic. ${ }^{[9]}$ The burden of reduced physical activity on mental health in childhood has increased over the years. The Department for Education and Health Education England seek new ways to promote the health and well-being of children. ${ }^{[11]}$ So far, there have been few studies that have tested yoga interventions in U.K. schools aged children. ${ }^{[26]}$

The Government's Green Paper 27 , Transforming Children and Young People's Mental Health Provision, focused on providing early intervention and prevention. The paper identified a need for interventions that can contribute towards the promotion of mental health and well-being. Due to the current COVID-19 pandemic, innovative and targeted interventions will be essential in supporting children during these difficult times. [27]

In addition, the amount of physical activity that children engage in has reduced, as $40 \%$ of parents said that their children receive the recommended minimum of 30 minutes of physical activity whilst at school. ${ }^{[9]}$ However, school closure means that these children may not be getting enough physical activity.

COVID-19 has many potential direct and indirect impacts on mental health. The Lancet Psychiatry published a position paper on mental health research priorities for the COVID-19 pandemic. [25] One of the research priorities was the populations' psychosocial effects related to COVID-19 and the need for rapid qualitative research with diverse people and communities to help to identify ways in which the adverse effects might be alleviated. Whilst out of school, children and young people are particularly vulnerable to worsening mental health.

\begin{abstract}
Aims
This project introduces research on the role of yoga, especially concerning public health issues of mental health and well-being in school-aged children in the U.K. The purpose of this project is to evaluate an existing yoga intervention designed in acrisis (Covid-19) to promote the mental health and well-being of school-aged children at home delivered online. It will seek to evaluate the yoga intervention developed by a U.K. based charity, Patanjali Yog Peeth Trust (PYPT). This research will provide an opportunity to evaluate a child-centred educational intervention that is aimed at children in the U.K. This research will highlight valuable information about the resources required to deliver such an intervention in the U.K. In addition, it will offer a better understanding of the use of yoga for children in the U.K.
\end{abstract}

The project will seek to promote alternative methods in managing mental health and well-being in school-aged children. Evidence suggests that yoga is an effective intervention in school-aged children; however, much of the evidence comes from underpowered studies and suffer from severe methodological flaws 30 .

\title{
The research questions.
}


The evaluation of the intervention will address:

1. Can yoga feasibly be implemented online for children?

2. Is online yoga effective at promoting mental health and enhancing school-aged children's well-being in the U.K.?

This study will allow the families of children to record their assessment of their child's mental health during the lockdown and their experiences of the yoga intervention during the COVID-19 pandemic. Parents/guardians of the participants who take part in the PYPT yoga will be interviewed. By investigating parents'/guardians' experiences and needs, I will understand the role of yoga intervention in promoting mental health and well-being among children in the U.K.

\section{Methodology}

The research will be systematically conducted in 2 phases.

\section{Systematic review of existing research on yoga in school-aged children}

Systematic reviews provide a framework/background to appropriately position new research activities and highlight any methodological flaws in published literature, including lack of detail on inclusion and exclusion criteria, insufficient synthesis of findings, and absent or inadequate assessment of the risk of bias included studies. To avoid duplication of systematic reviews, PROSPERO was searched for ongoing or recently completed systematic reviews. The systematic review protocol was prospectively registered on PROSPERO (CRD42020171943). [28] The systematic review will include RCT studies evaluating intervention processes. It aimed to compare the effectiveness of different interventions and estimate how much difference the intervention is likely to make if applied in practice. The findings of the included studies will be brought together in a narrative synthesis. The research can also provide a sense of the difficulties that can arise when delivering interventions that aim to achieve desired behaviours. The selection of the most appropriate design for a new research project can be very challenging. Therefore, it is vital to utilize past studies to act as guides to determine the best method of inquiry.

The review addresses the following questions:

1. Effect of yoga interventions on the mental health of school-aged children

2. Can yoga be used as an intervention to improve mental health in school children?

The author uses the PICOS framework to outline the review inclusion criteria:

- $\quad$ Population: Primary and secondary school children aged 5-16, anywhere mental health.

Intervention: School-based yoga intervention measuring the effects of yoga on

- Comparator: Any, including physical education (P.E.) or education as usual, or waitlist.

Outcomes: Depression (low mood), self-esteem, anger, stress or anxiety outcomes as either a primary or secondary outcome.

- $\quad$ Study design: - Randomised Control Trials (RCTs)

\section{Search strategy}

Searches will be performed using the Cumulative Index to Nursing and Allied Health Literature 
(CINAHL), PsycINFO, Education Resources Information Centre (ERIC), PubMed, Web of Science, Medline and Cochrane Library through Ovid. Results will be searched from January 2008 until Feb 2021; broadening the search criteria from January 2008 will capture the most RCTs possible.

The key words' yoga OR mindfulness - AND school AND Children OR child OR youth OR adolescent' will be used. The search is limited to studies in the English language. The word mindfulness is used to capture the most research articles possible, as some studies use mindfulness as a secondary outcome. No mental health search terms are used because preliminary searches suggest that study outcomes are diversely measured and use various language.

Table 1: Inclusion and exclusion criteria

\begin{tabular}{|c|c|c|}
\hline Eligibility Criteria & Inclusion & Exclusion \\
\hline Intervention & $\begin{array}{l}\text { Yoga practice that includes postures } \\
\text { and meditation evaluating mental } \\
\text { health (anxiety, low mood, depression) } \\
\text { in children }\end{array}$ & $\begin{array}{l}\text { Not using yoga as an } \\
\text { intervention or mindfulness } \\
\text { type only intervention }\end{array}$ \\
\hline Study Design & 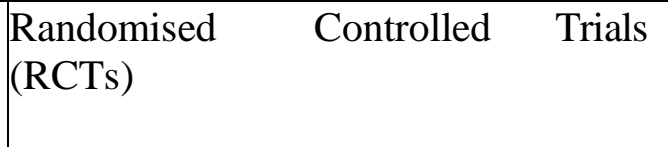 & $\begin{array}{l}\text { Prospective or retrospective } \\
\text { Cohorts, Pilots or feasibility } \\
\text { studies, Mixed method studies }\end{array}$ \\
\hline Setting & Mainstream school Setting & $\begin{array}{l}\text { Children in schools for Special } \\
\text { Educational Needs (SEN) }\end{array}$ \\
\hline Population & Children aged 5 years old and under 16 & $\begin{array}{l}\text { Children aged under } 5 \text { and } \\
\text { over } 16 \text { years }\end{array}$ \\
\hline Outcome & $\begin{array}{l}\text { Mental health outcomes using } \\
\text { validated clinical assessments or } \\
\text { parent/child/teacher self-report.' }\end{array}$ & $\begin{array}{l}\text { No mental health-related } \\
\text { outcomes reported }\end{array}$ \\
\hline Publication Date & January 2008 onwards & \\
\hline Language & English & Not in English \\
\hline Publication Status & Peer-reviewed papers & $\begin{array}{l}\text { Unpublished studies such as } \\
\text { records of ongoing research, } \\
\text { grey literature and theses }\end{array}$ \\
\hline
\end{tabular}

\section{Data Extraction}

Literature search results will be uploaded to EndNoteX9, which will serve as a record of screening against the selection criteria and used to remove duplicate results. Trials will be selected using the set inclusion/exclusion criteria in Table 1.

\section{Quality appraisal}

Version 2 of the Cochrane risk-of-bias tool for randomized trials ${ }^{[29]}$ will be used to summarise the results of the selected RCTs. Risk of bias will be assessed against the four domains in the Cochrane risk-of-bias tool: Randomization process, risk of bias due to deviations from the intended interventions, Missing outcome data, risk of bias in the measurement of the outcome and risk of bias in the selection of the reported result. Each criterion will be categorized as 'low', 'high' 
or 'some concerns' for each RCT study.

\section{Narrative synthesis of study findings}

The findings of studies of children with common mental health problems will be presented in table form. The findings of studies measuring expected mental health findings will be synthesized according to the trial comparator group, for example, yoga intervention to control group, because yoga was hypothesized to improve mental health outcomes than control groups. The findings will be grouped by those researchers reporting consistent findings, such as improvements or no change. Where studies report consistent findings, similarities between them in terms of setting, nature of intervention and outcome measures will be analyzed.

This is the first systematic review to have included only RCTs that focus solely on the effects of school-based yoga intervention on mental health outcomes. Previous systematic reviews have focused on a broader range of outcome measures and included a broad range of trials.

\section{Formative Phase: Evaluation of the yoga intervention}

The objective of this phase is to evaluate the online yoga intervention by PYPT for school-aged children. This phase will include qualitative interviews with parents of the participating children. Parents of children participating in PYPT yoga will be invited to an interview with the researcher and complete an online survey. Participants will be selected from the U.K. only. Participants will be informed about the study, and consent would be obtained from those willing to participate in the study. The results will be published in peer-reviewed journals, and recommendation will be made for future studies.

The yoga intervention is already currently being delivered to the community online via the online platform Zoom; all classes are delivered online due to Covid-19 restrictions. In line with government social distancing rules, all surveys and interviews will be conducted online. Approximately 60-70 children are participating voluntarily each week.

\section{Study Design and participants}

The study design is a mixed-methods approach with both quantitative and quantitative methods and conducted in two stages:

Stage 1. Parent and child characteristics and Strengths and Difficulties Questionnaire completed online via Opino

Stage 2. Structured interviews conducted online via Teams, WhatsApp or Zoom

Sample

A purposefully selected sample of parents/guardians, inclusion criteria:

- Adults ( $\geq 18$ years)

- Parents/guardians

- Participants can provide consent.

The exclusion criteria will be:

- Participants who are unwilling/unable to give consent to participate in the interview.

- Participants not living in the U.K. 


\section{Sample Size}

Parents will be approached by the PYPT yoga teacher and asked if they would like to participate in a research pilot. The PYPT charity will advertise the intended study on their home website and Facebook page. Participants will be selected from the U.K. via an advert. Participants will be informed about the study, and consent would be obtained from those willing to participate in the study. Consent forms will be sent to parents electronically via email. A representative sample of 15-20 parents/guardians will be purposefully selected for the formative phase of the study. For the qualitative interview, the author aims to recruit as many participants as possible, up to a maximum of 20 .

\section{Study procedure}

Parents will be approached by the PYPT yoga teacher and asked if they would like to participate in a research pilot using recruitment posters. PYPT charity will advertise the study on their home website, Facebook, Twitter page and individual WhatsApp groups across the country. Participants will be informed about the study, and consent obtained from those willing to participate in the study. Consent forms will be sent to parents electronically via WhatsApp.

Parents who consent to participate in the study will be asked to complete a parent and child characteristic questionnaire and the Strengths and Difficulties Questionnaire (SDQ) ${ }^{[30,31]}$ via Opinio online. A subsample will be interviewed using a structured interview schedule. The interview schedule for the study is developed specifically for parents. The framework of the interview schedule includes open-ended questions. During the interview, discussions around the child's overall health and well-being will be made, how long they have been practising yoga, and if they have noticed any changes.

\section{Analysis}

All analyses were carried out in SPSS (version 27.0). SDQ categories were calculated using syntax available from http://www.sdqinfo.com/py/sdqinfo/c0.py. Information obtained from the systematic review and the structured interview responses will be thematically organized to inform the effectiveness of the intervention in aiding the mental health and well-being of children.

\section{Dissemination, Outputs and anticipated Impact of the research}

It is intended that the research will provide an evidence base that will aid future studies and inform in developing yoga interventions that may aid in promoting child mental health and well-being. The study results will be published in peer-reviewed international journals; the researcher will also present the results at governmental conferences.

Depending on the acceptability and the feasibility of the intervention in the U.K. context, towards the end of the project, a funding application will be made to test the intervention in a randomized controlled trial as part of a postdoctoral fellowship.

\section{Ethical considerations}

The research will take place in the U.K. Applications for ethics approval will be submitted to the ethics committees at University College London. 


\section{Conclusion}

Due to the current COVID-19 pandemic, there has been a need for innovative and targeted interventions like the online yoga intervention that can support children during these difficult times. The intervention is unique; it is the first yoga intervention developed in a crisis (Covid-19) and delivered online free, and possibly the reason for many children participating. This study will aim to shape and inform policy resulting in a healthy society, reducing the burden of treating childhood illnesses like mental health. The research will help build a community of young adults who will have the skills to manage stress and build resilience.

\section{References}

1. Streeter, C.C., et al., Effects of yoga versus walking on mood, anxiety, and brain GABA levels: a randomized controlled MRS study. The Journal of Alternative and Complementary Medicine, 2010. 16(11): p. 1145-1152.

2. Stueck, M. and N. Gloeckner, Yoga for Children in the Mirror of the Science: Working Spectrum and Practice Fields of the Training of Relaxation with Elements of Yoga for Children. Early Child Development and Care, 2005. 175(4): p. 371-377.

3. PHE. Best start in life and beyond: Improving public health outcomes for children, young people and families Guidance to support the commissioning of the Healthy Child Programme 019: Health visiting and school nursing

services. 2018 [cited $2021 \quad 16$ Feburary ]; Available from: https://assets.publishing.service.gov.uk/government/uploads/system/uploads/ attachment data/file/686928/best start in life and beyond commissioning guidance 1.pdf.

4. Shribman, S. and K. Billingham. Healthy Child Programme - Pregnancy and the first five years. 2009 [cited 2020 July]; Available from: https://www.gov.uk/government/uploads/system/uploads/attachment data/file/ 167998/Health_Child_Programme.pdf

5. Nicholson, W. and P. Greenwood. Healthy child programme 0 to 19: health visitor andschool nurse commissioning. Public Health England. 2016 [cited 2021

February ]; Available from:

https://www.gov.uk/government/publications/healthy-child-programme-0-to- 19-health-visitorand-school-nurse-commissioning.

6. Sheridan, M.D., From Birth to Five Years Children's Developmental Progress. 3rd Edition ed. Vol. 3rd edition. 2014, London:: Routledge.

7. Smith, C.R., A. Katsiyannis, and J.B. Ryan, Challenges of serving students with emotional and behavioral disorders: Legal and policy considerations. Behavioral Disorders, 2011. 36(3): p. 185-194.

8. Kaley-Isley, L.C., et al., yoga as a complementary therapy for children and adolescents: a guide for clinicians. Psychiatry, 2010. 7(8): p. 20-32. 
9. Unicef. Children in lockdown: Rapid assessment of the impact of coronavirus on children in the U.K. 2020 [cited 202003 May]; Available from: https://www.unicef.org.uk/wpcontent/uploads/2020/04/UnicefUK_ChildrenInLockdown_RapidAssessment.p df.

10. Perrin, J.M., M.T. Stein, and R.W. Amler, Clinical practice guideline: treatment of the school-aged child with attention-deficit/hyperactivity disorder. Paediatrics,, 2001. 4(108): p. 1033-1044.

11. Department of Health. Future in mind Promoting, protecting and improving our children and young people's mental health and well-being. 2015 [cited 202001 May]; Available from:

https://assets.publishing.service.gov.uk/government/uploads/system/uploads/ attachment_data/file/414024/Childrens_Mental_Health.pdf.

12. Butzer, B., et al., Effects of a Classroom-Based Yoga Intervention on Cortisol and Behavior in Second- and Third-Grade Students: A Pilot Study. Journal of Evidence-Based Complementary \& Alternative Medicine, 2015. 20(1): p. 41-49.

13. Neumann, D., Prenatal Yoga: The Right Choice for Mother and Baby. Honours Projects. . Honors Projects., 2011. 89.

14. Satyapriya, M., et al., Effect of integrated yoga on stress and heart rate variability in pregnant women. International Journal of Gynecology \& Obstetrics, 2009. 104(3): p. 218-222.

15. Yoo, Y.-G., et al., The effects of mind subtraction meditation on depression, social anxiety, aggression, and salivary cortisol levels of elementary school children in South Korea. Journal of pediatric nursing, 2016.31(3): p. e185-e197.

16. Pramanik, T., et al., Immediate effect of slow pace bhastrika pranayama on blood pressure and heart rate. The Journal of Alternative and Complementary Medicine, 2009. 15(3): p. 293-295.

17. Epple, A., Infant massage for children with disabilities and special needs. The practising midwife, 2011. 14(2): p. 30, 32-30, 32.

18. CDC. Playground safety-United States. . 2016 [cited 202001 May]; Available from: https://www.cdc.gov/safechild/playground/index.html

19. Bazzano, A.N., et al., Effect of mindfulness and yoga on quality of life for elementary school students and teachers: results of a randomized controlled school-based study. Psychology research and behavior management, 2018. 11: p. 81-89.

20. Shreve, M., et al., Using Yoga to Reduce Anxiety in Children: Exploring School-Based Yoga Among Rural Third- and Fourth-Grade Students. Journal of Pediatric Healthcare, 2021. 35(1): p. 42-52.

21. WHO. Physical Activity and Young People. $2015 \quad$ [cited 202001 May ]; Available from: http://www.who.int/dietphysicalactivity/factsheet_young_people/en/

22. NHSDigital. Mental Health of Children and Young People in England, 2017 (PAS). 
2018; $\quad$ Available from: $\quad$ https://digital.nhs.uk/data-and-

information/publications/statistical/mental-health-of-children-and-young- people-inengland/2017/2017.

23. Obeng, C.S., Physical activity lessons in preschools. Journal of Research in Childhood Education, 2009. 24(1): p. 50-59.

24. Newlove-Delgado, T., et al., Child mental health in England before and during the COVID-19 lockdown. The Lancet Psychiatry, 2021.

25. ONS. Coronavirus (COVID-19) related deaths by ethnic group, England and Wales 2 March 2020 to 10 April 2020. 2020 [cited 202001 December]; Available from:

https://www.ons.gov.uk/peoplepopulationandcommunity/birthsdeathsandmarri

ages/deaths/articles/coronavirusrelateddeathsbyethnicgroupenglandandwales

12march2020to10april2020.

26. James-Palmer, A., et al., Yoga as an Intervention for the Reduction of Symptoms of Anxiety and Depression in Children and Adolescents: A Systematic Review. Frontiers in Pediatrics, 2020. 8(78).

27. Lancet, T., Child mental health services in England: a continuing crisis. The Lancet, 2020. 395(10222): p. 389.

28. Khunti, K., et al. The effects of yoga on mental health in school aged children: a systematic review and narrative synthesis of randomized control trials. PROSPERO: International prospective register of systematic reviews 2020; Available from: https://www.crd.york.ac.uk/prospero/display_record.php?RecordID=171943.

29. Sterne, J.A., et al., RoB 2: a revised tool for assessing risk ofbias in randomized trials. bmj, 2019. 366.

30. Goodman, R., The Strengths and Difficulties Questionnaire: a research note. Journal of child psychology and psychiatry, 1997. 38(5): p. 581-586.

31. Goodman, R., The extended version of the Strengths and Difficulties Questionnaire as a guide to child psychiatric caseness and consequent burden. Journal of child psychology and psychiatry, 1999. 40(5): p. 791-799. 